\title{
Epidemiology of neuroendocrine tumors of the appendix in the USA: a population-based national study (2014-2019)
}

\author{
Motasem Alkhayyat a, Mohannad Abou Saleh ${ }^{b}$, Wendy Coronado a, Mohammad Abureeshc, \\ Mohammad Zmailia, Thabet Qapaja ${ }^{a}$, Ashraf Almomani ${ }^{\mathrm{a}}$, George Khoudarid, Emad Mansoor ${ }^{\mathrm{e}}$, \\ Gregory Coopere
}

Cleveland Clinic Foundation, Ohio; Staten Island University Hospital, NY; University Hospitals Cleveland Medical

Center, Ohio, USA

\section{Abstract}

\begin{abstract}
Background The appendix is the third most common place for neuroendocrine tumors (NETs) along the digestive tract and NETs are the most common neoplasms of the appendix. However, there are limited population-based data on the epidemiology of this disease. Using a large database, we sought to describe the epidemiology and risk association of NETs of the appendix.
\end{abstract}

Methods We queried a multi-institutional database (Explorys Inc., Cleveland, OH, USA), comprising 360 hospitals in the United States (US), for patients with a diagnosis of NETs of the appendix from 2014-2019.

Results Of the 30,324,050 individuals in the database, 2020 patients had an appendiceal NET diagnosis $(0.007 \%)$. The most common presenting symptoms included abdominal pain, nausea, vomiting and diarrhea. Patients with appendiceal NETs were more likely to be female (odds ratio [OR] 1.36, 95\% confidence interval [CI] 1.24-1.49), Caucasian (OR 2.71, 95\%CI 2.40-3.07), with a history of smoking (OR 1.82, 95\%CI 1.65-2.01), family history of primary gastrointestinal malignancy (OR 7.26, 95\%CI 6.31-8.33), diagnosis of multiple endocrine tumor type 1 (OR 52.31, 95\%CI 23.15-118.23), or neurofibromatosis type 1 (OR 16.37, 95\%CI 7.24-37.01).

Conclusions In a population-based study in the US, using the Explorys database, we found the overall prevalence of NETs of the appendix to be 7 per 100,000 persons. The incidence in the year January 2019-January 2020 was 0.4 per 100,000 individuals. These rates are higher than previously reported and may be more accurate, given the more comprehensive nature of the Explorys database.

Keywords Neuroendocrine tumor, appendix, epidemiology, risk factors

Ann Gastroenterol 2021; 34 (1): 1-8 anternal Medicine Department, Cleveland Clinic Foundation, Cleveland, Ohio (Motasem Alkhayyat, Wendy Coronado, Mohammad Zmaili, Thabet Qapaja, Ashraf Almomani); ${ }^{\mathrm{b}}$ Gastroenterology, Hepatology, and Nutrition, Cleveland Clinic Foundation, Cleveland, Ohio (Mohannad Abou Saleh); ' Internal Medicine Department, Staten Island University Hospital, Staten Island, NY (Mohammad Abureesh); ${ }^{\mathrm{d} D}$ Department of Hospital Medicine, Cleveland Clinic Foundation, Cleveland, Ohio (George Khoudari); ${ }^{\mathrm{e}}$ Gastroenterology and Hepatology, University Hospitals Cleveland Medical Center, Cleveland, Ohio (Emad Mansoor, Gregory Cooper), USA

Conflict of Interest: None

Correspondence to: Motasem Alkhayyat, MD, Department of Internal Medicine, Cleveland Clinic Foundation, 9500 Euclid Ave, Cleveland, OH 44195, USA, e-mail: motasemalkhayyat@gmail.com

Grant Support: Supported in part by the Case Comprehensive Cancer Center (P30CA43703) and Cleveland Digestive Disease Research Core Center (P30DK097948)

Received 16 November 2020; accepted 5 April 2021; published online 14 June 2021

DOI: https://doi.org/10.20524/aog.2021.0643

\section{Introduction}

Gastroenteropancreatic neuroendocrine tumors (NETs) is an inclusive term referring to NETs of the gastrointestinal (GI) system. Gastroenteropancreatic NETs are commonly divided into pancreatic NETs and luminal carcinoid tumors, also known as luminal NETs [1]. As carcinoid tumors arise from neuroendocrine cells along the GI tract, they can also occur within the appendix [2]. NETs of the appendix have features that differentiate this type from other NETs in the rest of the GI tract. These neoplasms are more frequently benign and usually occur at a younger age. They are also associated with the expression of S-100 [3]. NETs are frequently distributed in the distal third of the appendix and can result in luminal obstruction. Diagnosis is generally made following an appendectomy and histological examination of the appendix [4]. Although most NETs of the appendix (aNETs) are small, asymptomatic and slow-growing, they can be aggressive, invasive, and metastatic [5]. 
Because appendiceal tumors and NETs are uncommon, research has been limited to single-institution data. The existing body of population studies has largely examined data from the 1980s-2000s [5,6]. A recent population-based study showed a 6.4-fold increase in age-adjusted incidence rates of NETs between 1973 and 2012 [6]. Previous epidemiological data showed that aNETs were the most frequent NETs along the GI tract; however, their relative frequency has decreased as a result of a concomitant overall increase in other types of NETs [7]. Given the inconsistent incidence identified in smaller studies and the rate of change in large population-based studies, an updated investigation to clarify the demographic risk factors and current prevalence is needed. In this study, we also investigated clinically relevant symptoms associated with NETs.

\section{Materials and methods}

\section{Database}

We performed a retrospective cohort analysis using a multiple health system data analytics and research platform (Explorys Inc., Cleveland, OH, USA), developed and prospectively maintained by IBM Corporation, Watson Health [8]. Explorys captures data from the electronic health records of a total of 360 hospitals from 26 healthcare systems in the United States (US). Data from more than 50 million unique patients, representing approximately $15 \%$ of the population across all 50 states, are captured and thus provide a broad regional distribution of population. The "Systematized Nomenclature Of Medicine - Clinical Terms" (SNOMEDCT) hierarchy is used to arrange diagnoses, findings and procedures [9], while SNOMED and RxNorm are used to code for drug prescriptions [10]. Access to Explorys is granted to participating healthcare systems. Informed consent was waived since patient data are de-identified. In order to protect patient confidentiality, the database rounds cell counts to the nearest 10. It is worth highlighting that the Explorys database has been used in multiple medical fields, including gastroenterology [11-15].

\section{Patient selection}

Using the Explorys platform, we identified a cohort of patients diagnosed with aNETs during the period of December 2014 and December 2019. We also identified a cohort of patients with a first-ever aNET diagnosis during the year from January 2019 to January 2020.

\section{Risk factors, predisposing medical conditions, and GI signs and symptoms}

Using SNOMED-CT codes, we identified possible risk factors, predisposing medical conditions, and associated GI signs and symptoms of aNETs suggested by prior literature. Possible risk factors and predisposing medical conditions included alcohol abuse, smoking, diabetes mellitus, obesity, family history of GI cancer, multiple endocrine neoplasia type 1 syndrome (MEN type 1), neurofibromatosis type 1 (NF type 1), Crohn's disease (CD), and ulcerative colitis (UC). Signs and symptoms included abdominal pain, diarrhea, nausea, vomiting, flushing, GI bleeding, obstruction, perforation, acute appendicitis, intussusception, and volvulus. Given the cross-sectional nature of the study, a temporal relationship with signs and symptoms was difficult to establish.

\section{Statistical analysis}

Demographics and associated diseases of our patient cohort were characterized by descriptive statistics. To calculate the overall period prevalence, we divided the total number of individuals with aNET by the total number of individuals in Explorys (2014-2019). The rate of new cases of aNET in the US in the year 2019-2020 was obtained by dividing the number of individuals with a first-ever aNET diagnosis from January 2019 through January 2020 by the total number of individuals in Explorys in the past year, in an attempt to provide a proxy estimate of annual incidence. The odds ratio (OR) for univariate analysis, its standard error and 95\% confidence interval (CI) were calculated using the MedCalc Statistical Software with a case-control design (MedCalc Version 19.1.6). To adjust for confounding factors, we constructed a multivariate regression analysis model using the Statistical Package for Social Sciences (SPSS version 25, IBM Corp.). Variables adjusted for were age, sex, race and multiple comorbidities, including tobacco smoking, alcohol abuse, diabetes mellitus, obesity (body mass index $>30$ ), family history of GI malignancy, MEN type 1 syndrome, and NF type 1 syndrome. A P-value $<0.05$ was considered statistically significant.

\section{Results}

A total of $30,324,050$ individuals above 18 years old in the database between 2014 and 2019 composed the source population. Of these, $2020(0.007 \%)$ patients had a SNOMED-CT diagnosis of aNET and represented the study group. The baseline characteristics of the patients and the control group are presented in Table 1. The incidence of new cases of aNET in (2019-2020) was 0.4 per 100,000 individuals.

\section{Interval epidemiology of aNETs}

aNETs accounted for $0.2 \%$ of all NETs. Of the 2020 patients with aNET, the majority were Caucasian 
(84.4\%) and adults (18-65 years of age) (71.7\%) (Table 1). The overall 5-year prevalence was 7 per 100,000 individuals. The prevalence was highest in age group 75-79 $(9.4 / 100,000)$ (Fig. 1). Caucasians had the highest prevalence $(8.8 / 100,000)$

Table 1 Baseline patient characteristics of aNET and control groups

\begin{tabular}{|c|c|c|}
\hline Characteristics & $\begin{array}{l}\text { aNETs } \\
\begin{array}{l}\mathrm{N}=2020 \\
(\%)\end{array}\end{array}$ & $\begin{array}{c}\text { Non-aNETs } \\
\mathrm{N}=30,322,020 \\
(\%)\end{array}$ \\
\hline \multicolumn{3}{|l|}{ Age } \\
\hline $20-24$ & $70(3.5)$ & $2,018,690(6.7)$ \\
\hline $25-29$ & $110(5.4)$ & $2,370,450(7.8)$ \\
\hline $30-34$ & $150(7.4)$ & $2,379,730(7.8)$ \\
\hline $35-39$ & $140(6.9)$ & $2,335,990(7.7)$ \\
\hline $40-44$ & $120(5.9)$ & $2,160,570(7.1)$ \\
\hline $45-49$ & $150(7.4)$ & $2,225,140(7.3)$ \\
\hline $50-54$ & $190(9.4)$ & $2,311,150(7.6)$ \\
\hline $55-59$ & $220(10.9)$ & $2,589,580(8.5)$ \\
\hline $60-64$ & $220(10.9)$ & $2,564,930(8.5)$ \\
\hline $65-69$ & $210(10.4)$ & $2,338,080(7.7)$ \\
\hline $70-74$ & $170(8.4)$ & $2,002,480(6.6)$ \\
\hline $75-79$ & $140(6.9)$ & $1,493,860(4.9)$ \\
\hline $80-84$ & $80(4.0)$ & $1,094,580(3.6)$ \\
\hline $85-90$ & $30(1.5)$ & $810,570(2.7)$ \\
\hline $90+$ & $20(1.0)$ & $810,570(2.7)$ \\
\hline \multicolumn{3}{|l|}{ Sex } \\
\hline Male & $733(36)$ & $12,954,076(43)$ \\
\hline Female & $1287(64)$ & $17,367,944(57)$ \\
\hline \multicolumn{3}{|l|}{ Race } \\
\hline Caucasian & $1705(84.4)$ & $19,349,990(63.8)$ \\
\hline African American & $153(7.6)$ & $3,426,260(11.3)$ \\
\hline Asian & $17(0.8)$ & $505,494(1.7)$ \\
\hline \multicolumn{3}{|l|}{ Comorbidities } \\
\hline Tobacco use & $510(25.2)$ & $4,539,110(15.0)$ \\
\hline Alcohol abuse & $70(3.5)$ & $667,330(2.2)$ \\
\hline Hypertension & $860(42.6)$ & $8,671,060(28.6)$ \\
\hline Diabetes mellitus & $330(16.3)$ & $3,673,800(12.1)$ \\
\hline Metabolic syndrome $\mathrm{x}$ & $30(1.5)$ & $164,380(0.5)$ \\
\hline Obese & $330(16.3)$ & $2,008,960(6.6)$ \\
\hline primary GI cancer & $690(34.2)$ & $201,130(0.7)$ \\
\hline $\begin{array}{l}\text { Family history of GI } \\
\text { cancer }\end{array}$ & $110(5.4)$ & $426,890(1.4)$ \\
\hline MEN 1 & $5(0.2)$ & $820(0.003)$ \\
\hline NF 1 & $5(0.2)$ & $3,710(0.01)$ \\
\hline Ulcerative colitis & $34(1.7)$ & $139769(0.5)$ \\
\hline Crohn's disease & $51(2.5)$ & $163212(0.5)$ \\
\hline
\end{tabular}

aNETs, appendiceal neuroendocrine tumors; GI, gastrointestinal; MEN, multiple endocrine neoplasm; NF, neurofibromatosis compared to African Americans (4.5/100,000) and Asians $(0.1 / 100,000)$. The prevalence was higher in females $(7.4 / 100,000)$ compared to males $(5.7 / 100,000)$. The prevalence of patients who developed carcinoid syndrome was $8.2 \%$, while $35.3 \%$ had appendicitis. Right-sided valve disease was present in 10 of the patients who had carcinoid syndrome. Among 550 patients checked for chromogranin A, $190(34.5 \%)$ had an elevated level, while among 470 checked for 5-hydroxyindoleacetic acid, 110 (23.4\%) patients tested positive.

\section{Univariate analysis for risk factors and predisposing medical conditions}

Patients with aNET were more likely to be Caucasian and female (Table 2). Patients who developed aNET were more likely to have a history of smoking and alcohol abuse. In terms of predisposing medical conditions, individuals with aNET were more likely to have a diagnosis of obesity, diabetes mellitus, UC, CD, MEN type 1, NF type 1, and family history of GI cancer (Table 2).

\section{Multivariate analysis for risk factors and predisposing medical conditions}

In a multivariate regression analysis model to adjust for confounding factors, there was no difference in the risk of aNET between adults (18-65 years) and elderly patients (age $>65$ years). However, patients who developed aNET were more likely to be female (OR 1.36, 95\%CI 1.24-1.49), Caucasian (OR $2.71,95 \%$ CI 2.40-3.07), with a history of smoking (OR 1.82, $95 \%$ CI 1.65-2.01), family history of primary GI malignancy

Table 2 Risk factors for neuroendocrine tumors of the appendix using univariate analysis

\begin{tabular}{lccc}
\hline Risk factors & OR & $95 \% \mathrm{CI}$ & P-value \\
\hline Age $>65$ years old & 1.12 & $1.01-1.23$ & 0.0246 \\
Female sex & 1.30 & $1.19-1.42$ & $<0.001$ \\
Caucasian race & 3.35 & $2.95-3.81$ & $<0.001$ \\
Alcohol abuse & 1.58 & $1.25-2.01$ & $<0.001$ \\
Smoking & 1.91 & $1.69-2.15$ & $<0.001$ \\
Obesity & 2.81 & $2.50-3.16$ & $<0.001$ \\
\hline Diabetes mellitus & 1.41 & $1.25-1.59$ & $<0.001$ \\
Family history of primary GI & 3.99 & $3.30-4.84$ & $<0.001$ \\
cancer & & & \\
\hline MEN type 1 & 90.56 & $37.55-218.38$ & $<0.001$ \\
NF type 1 & 20.01 & $8.32-48.16$ & $<0.001$ \\
\hline Ulcerative colitis & 4.29 & $3.14-5.87$ & $<0.001$ \\
\hline Crohn's disease & 4.62 & $3.49-6.11$ & $<0.001$ \\
\hline $\begin{array}{l}\text { OR odds ratio; CI, confidence interval; GI, gastrointestinal; MEN, multiple } \\
\text { endocrine neoplasm; NF, neurofibromatosis }\end{array}$ & & & \\
\hline
\end{tabular}




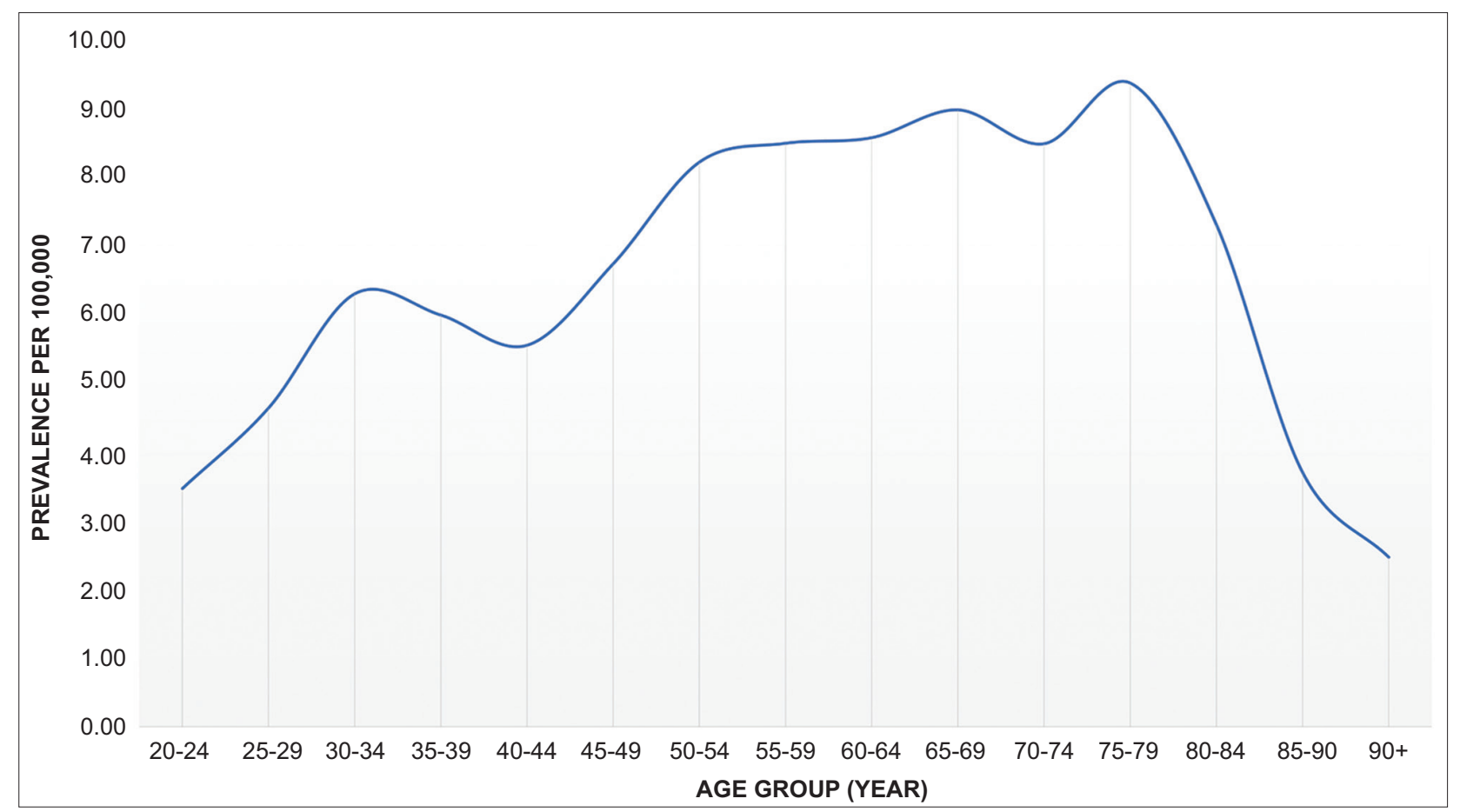

Figure 1 Prevalence of neuroendocrine tumors of the appendix among different age-groups per 100,000 population

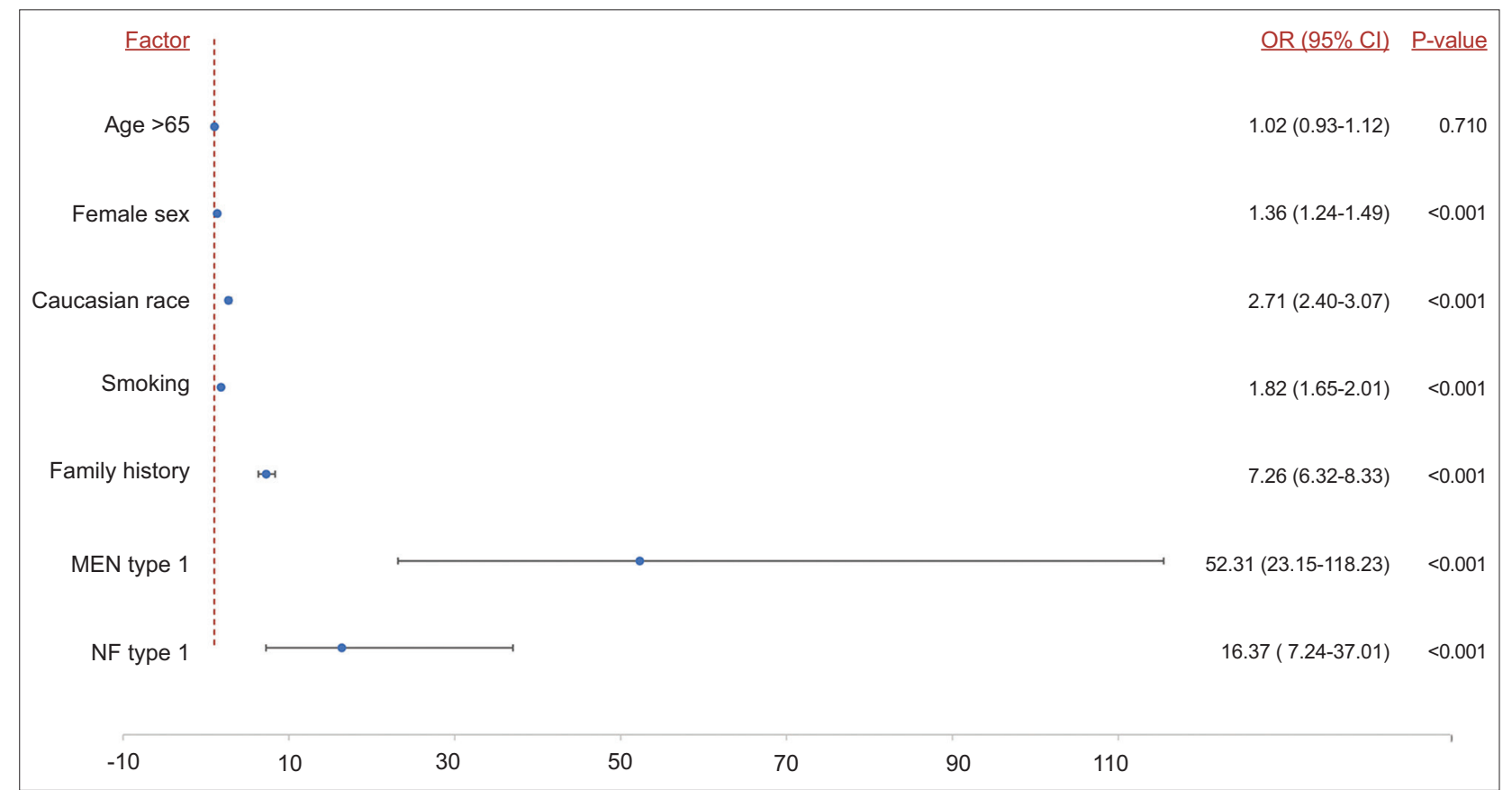

Figure 2 Risk factors associated with development of neuroendocrine tumors of the appendix in multivariate analysis aNETs, neuroendocrine tumors of the appendix; GI, gastrointestinal; MEN, multiple endocrine neoplasm; NF, neurofibromatosis

(OR 7.26, 95\%CI 6.31-8.33), diagnosis of MEN type 1 (OR 52.31, 95\%CI 23.15-118.23), or NF type 1 (OR 16.37, 95\%CI 7.24-37.01) (Fig. 2).

\section{Associated GI signs and symptoms}

Patients diagnosed with aNET were more likely to exhibit nausea, vomiting, abdominal pain, diarrhea, and flushing. 
They were also more likely to have abdominal obstruction, GI bleeding, acute appendicitis, GI perforation, intussusception, and volvulus (Fig. 3).

\section{Discussion}

In this study of 2020 patients with aNETs, we found that the greatest distribution of aNET patients occurred in the age range of 50-80 years old. Common symptoms associated with aNET included abdominal pain, acute appendicitis, and nausea. Significant risk factors for aNET were MEN type 1, NF type 1, family history of primary GI cancer, Caucasian race, smoking, and female sex.

The majority of the available reported incidence rates of aNETs are based on the Surveillance, Epidemiology, and End Results (SEER) program. In the analysis of the SEER database between 1973 and 1997, Maggard et al. found a relatively stable age-adjusted incidence of aNETs between 1973 (1.2 per million) and 1997 (2.1 per million) compared to other sites [7]. There was a rise in the incidence of rectal, gastric and small bowel carcinoids, with a concomitant decrease in the percentage of appendiceal carcinoid tumors. More specifically, $31.8 \%$ of all carcinoids were located in the appendix between the period 1973 to 1979 , compared with $12.0 \%$ in the period of 1990-1997 [7]. Subsequent analysis of the same database between 1973 and 2003 showed an ageadjusted incidence of 0.58 per $1,000,000$ people per year [5]. In the most recent analysis from the SEER database, for the period between 2000 and 2009, Marmor et al. reported annual incidences of aNETs of 0.17 and 0.27 per 100,000 individuals in 2000 and 2009, respectively [16]. The decrease in ageadjusted incidence is perhaps attributable to the fact that incidental appendectomies were more commonly done in the past. In our study, we found that the overall incidence between January 2019 and January 2020 was 0.4 per 100,000, higher than previously reported. While this might be attributed to the differences in databases, as the SEER program includes only malignant forms of carcinoid tumors, it may also reflect a rise in the incidence of aNETs $[5,6]$.

Large population studies evaluating trends in the incidence of aNETs during the period 1983-1998 identified a higher incidence in younger individuals: 15-19 years of age in women and 20-29 years of age in men [17]. In a review by Goede et al., the average age for aNET diagnosis was in the fourth decade of life [4], and in another large study with 11,427 carcinoid tumors the average age of patients with aNETs was 54.4 years [7]. Our data revealed a greater prevalence in the 50-80 age range. These differences could be a result of shifting incidence rates across decades, as the current population studies concerning carcinoid tumors have largely been based on data from the 1980s-2000s. Given the evidence of incidence changes reported in the 2000s, there may have been changes in the prevalence across the past 2 decades. The prevalence of aNETs in review articles is reported to be $0.2-0.9 \%$ in patients undergoing appendectomy $[3,4]$. The $20-$ year prevalence of aNETs between 1993-2012, based on the SEER database, was 2 per 100,000 persons [6]. In our study, we found a higher period prevalence of 7 per 100,000 individuals.

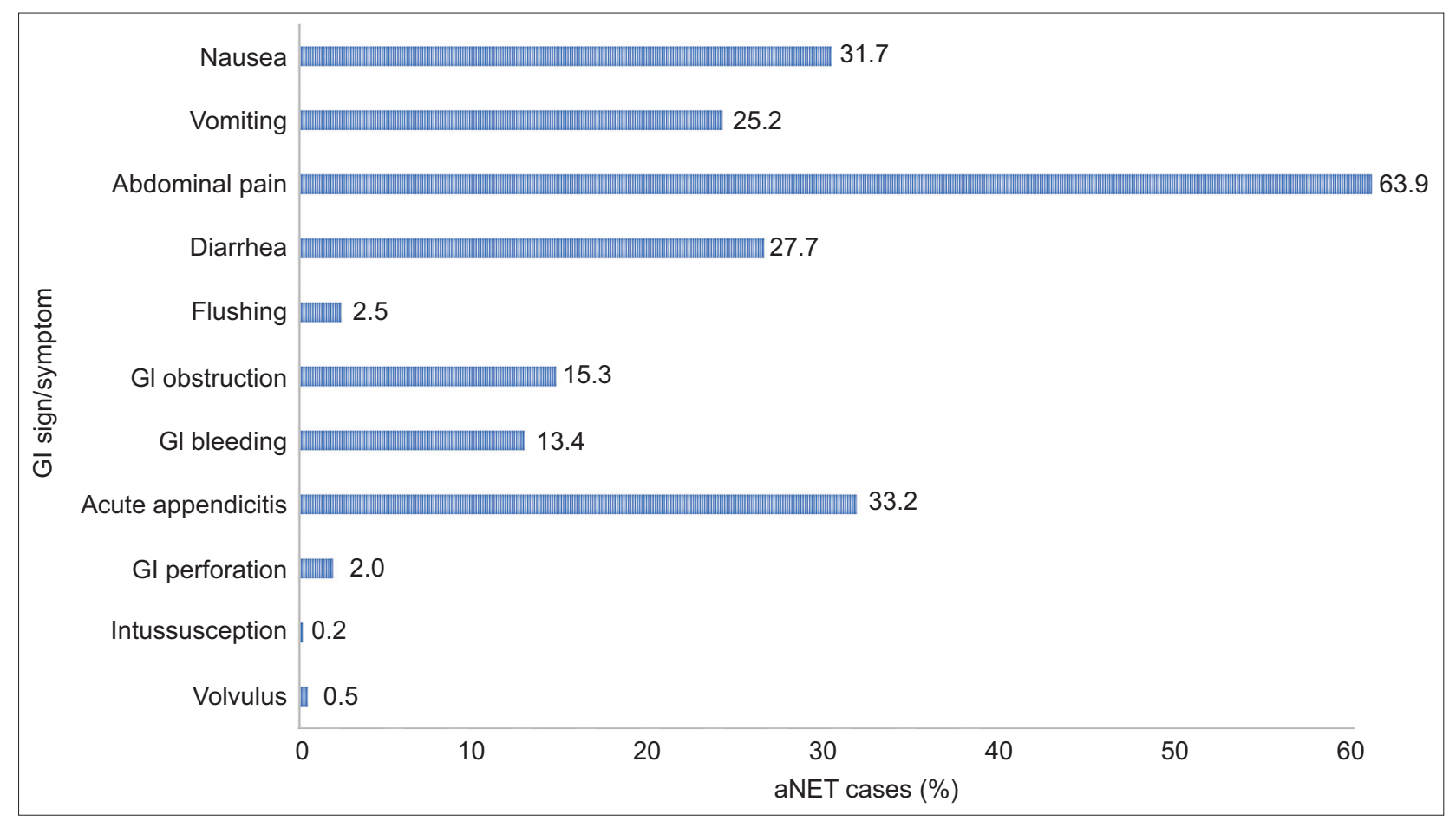

Figure 3 Signs and symptoms associated with aNETs

GI, gastrointestinal; aNET, appendiceal neuroendocrine tumor 
As mentioned previously, this may reflect a rising prevalence, but the difference might also be related to the inclusion of only malignant forms of carcinoid tumors in the SEER program.

Comparing the prevalence of aNETs in other countries, a prospective study in Austria over a one-year period, performed by Niederle et al., reported a rate of 0.08 per 100,000 malignant aNETs in 2004 [18]. Taal et al. reported the age-standardized incidence of NETs to be 1.8 and 1.9 per 100,000 for Dutch males and females, respectively, among which aNETs accounted for $27 \%$ of total cases [19]. Comparable incidences were reported in a study conducted in Sweden, with 0.4 and 0.8 per 100,000 for males and females, respectively [17]. In comparison, Ploeckinger et al. demonstrated that aNETs accounted for 3.2\% in a cohort of 1236 patients in Germany [20], similar to a Taiwanese study that reported a prevalence of $3.6 \%$ of aNETs among a cohort of 2187 NETs [21]. A slightly higher prevalence was reported in Argentina in a study conducted by O'Connor et al., who reported a prevalence of $7.6 \%$ of aNETs among a cohort of 461 patients with gastroenteropancreatic NET [22]. A recent Turkish study showed that aNETs account for $0.39 \%$ of total appendectomies, consistent with the prior literature (0.2-0.7\%) and comparable to the prevalence in our study ( $0.2 \%$ of total NETs) [23].

Several articles have noted that the incidence of aNETs is higher in the female population, which may be an effect of higher appendectomy rates in younger women [4,5,7]. Analysis of racial distribution showed that Caucasian patients account for the majority of cases of aNETs: $87 \%$ of the cases in SEER analysis between 1988 and 2003. In our study, we found a higher rate of developing aNETs among females compared to males $(7.4$ vs. $5.7 / 100,000)$ and a higher rate in Caucasian $(8.8 / 100,000)$ compared to other races. Based on the multivariate analysis model, we found female sex and Caucasian race are more likely to be associated with aNETs.

Risk factors and associated medical comorbidities are not commonly discussed in the literature, probably because NETs are incidental findings. There are few studies that have explored the association between hypertension, smoking and developing aNETs. The primary discussion in the literature lies with carcinoid syndrome and its effect on the heart due to the secretion of serotonin when tumors have invaded the liver. In these cases, blood pressure can be labile, with hypertension or hypotension. The rate of hypertension in cancer patients is similar to that in the general population (29\%); however, the prevalence is higher in patients who have undergone chemotherapy (37\%) [24]. Given that the prevalence of hypertension is elevated compared to the general population, further research is needed to understand its significance in patients with NETs. Tobacco smoking is a well-known risk factor for multiple neoplasms; however, little is known about its effect on the development of NETs. A few small studies showed an approximately twofold greater risk of developing GI NETs $[25,26]$. In a larger case-control study, Rinzivillo et al. demonstrated a proportional positive association between the amount of smoking and the risk of small bowel NETs [27]; however, other studies did not reach the same conclusion [28]. In our study, smoking was more strongly associated with aNETs, even after adjusting for genetic syndromes and family history of primary GI malignancy. Obesity and diabetes are established risk factors for the development of NETs, and this is consistent with our findings [29]. Furthermore, we found that patients with aNETs are more likely to have other GI malignant tumors. The literature is limited in regard to the association of aNET with extra-appendiceal GI cancers.

A previous study of risk factors associated with NETs identified a family history of neoplasm [28]. In this study, we also found significantly greater odds of aNET associated with a family history of GI cancer, after adjusting for multiple confounders. Furthermore, a family history of carcinoid tumors was found to be associated with a greater risk of developing NETs, as well as other cancers [30]. It is known that NETs have some association with MEN type 1 syndrome, and this was demonstrated in the current study [17]. NF type 1 is linked to a higher risk of pancreatic and luminal NETs, more prominently reported in the ampulla of Vater as well as the appendix [31], consistent with our findings.

Inflammatory bowel disease (IBD), including UC and $\mathrm{CD}$, is well-known to predispose to the development of adenocarcinoma of the colon [32]. Several reports linked IBD to a greater risk of NETs, based on the finding of elevated numbers of neuroendocrine cells in inflamed mucosa. It has been suggested that long-standing inflammation is directly responsible for the development of NETs, in a similar fashion to the underlying pathophysiology of colorectal neoplasm [33-35]. In a prospective study conducted in Spain, which included 590 patients with IBD followed for 7 years, 80 patients developed different cancers. The relative risk for developing NETs in patients with IBD was 13.1 (95\%CI 1.8229.7) [36]. Using a univariate analysis model, we found that patients with IBD are at higher risk of developing aNETs. The risk was comparable between CD and UC patients.

There are a few limitations to this study that largely stem from the nature of the database. Data entry and classification can be a source of potential bias and may influence the true estimates of diseases. Unfortunately, patients' information in the database is de-identified and pathology results cannot be verified for each corresponding patient, while survival data are also not available in Explorys. However, it is important to note that, compared to ICD coding, SNOMED-CT allows for more concepts to be coded per clinical document, making it more accurate in terms of documenting diagnoses and pertinent patient information. Moreover, Explorys has been validated and used in multiple specialties, including gastroenterology [37-39]. Despite the aforementioned limitations, it is worth highlighting that this is the largest and one of the few studies to date to evaluate the epidemiology, and risk factors of patients with aNETs in the US population. In comparison to the SEER database, which includes only malignant pathologies, Explorys captures both benign and malignant pathologies. The data also provide a much-needed update to the existing body of population studies, which has largely focused on data from the 1980s-2000s. We also report symptoms associated with the aNET population, not commonly identified in the literature. Using multivariate analysis, several steps were implemented to avoid confounding bias.

In conclusion, this is the largest study to date utilizing a non-SEER database to evaluate the epidemiology, risk factors, 
predisposing medical conditions and GI symptoms associated with the development of aNETs. We found a significantly higher prevalence and incidence rates of aNETs than previously found in the literature. Additionally, our multivariate analysis determined multiple risk factors associated with aNET development. However, further research is needed to elucidate the potential mechanisms for the role of the risk factors and predisposing medical conditions identified in our study.

\section{Summary Box}

\section{What is already known:}

- Gastroenteropancreatic neuroendocrine tumors (NETs) are divided into pancreatic NETs and luminal carcinoid tumors

- The majority of epidemiological studies are based on the Surveillance, Epidemiology, and End Results database

\section{What the new findings are:}

- The overall prevalence of NET of the appendix (aNET) was 7 per 100,000 persons, while the incidence during a single year (January 2019 to January 2020) was 0.4 per 100,000 individuals

- Common symptoms associated with aNET included abdominal pain, acute appendicitis and nausea

- Significant risk factors for aNET were multiple endocrine neoplasia type 1 , neurofibromatosis type 1 , family history of primary gastrointestinal cancer, Caucasian race, smoking, and female sex

\section{References}

1. Gluckman CR, Metz DC. Gastric neuroendocrine tumors (carcinoids). Curr Gastroenterol Rep 2019;21:13.

2. Tchana-Sato V, Detry O, Polus M, et al. Carcinoid tumor of the appendix: a consecutive series from 1237 appendectomies. World J Gastroenterol 2006;12:6699-6701.

3. Carr NJ, Sobin LH. Neuroendocrine tumors of the appendix. Semin Diagn Pathol 2004;21:108-119.

4. Goede AC, Caplin ME, Winslet MC. Carcinoid tumour of the appendix. Br J Surg 2003;90:1317-1322.

5. Mullen JT, Savarese DM. Carcinoid tumors of the appendix: a population-based study. J Surg Oncol 2011;104:41-44.

6. Dasari A, Shen C, Halperin D, et al. Trends in the incidence, prevalence, and survival outcomes in patients with neuroendocrine tumors in the United States. JAMA Oncol 2017;3:1335-1342.

7. Maggard MA, O'Connell JB, Ko CY. Updated population-based review of carcinoid tumors. Ann Surg 2004;240:117-122.

8. IBM Corporation. The IBM Explorys Platform: liberate your healthcare data. Available from: https://www.ibm.com/downloads/ cas/4P0QB9JN [Accessed 13 May 2021].
9. US National Library of Medicine Unified Medical Language System (UMLS). Systematized Nomenclature of MedicineClinical Terms (SNOMED CT). Available from: https://www.nlm. nih.gov/research/umls/Snomed/snomed_main.html] [Accessed 13 May 2021].

10. Nelson SJ, Zeng K, Kilbourne J, Powell T, Moore R. Normalized names for clinical drugs: RxNorm at 6 years. J Am Med Inform Assoc 2011;18:441-448.

11. Al-Kindi SG, Oliveira GH. Prevalence of preexisting cardiovascular disease in patients with different types of cancer: the unmet need for onco-cardiology. Mayo Clin Proc 2016;91:81-83.

12. Mahadevan U, Martin CF, Dubinsky M, Kane SV, Sands BE, Sandborn W. 960 Exposure to anti-TNF therapy in the third trimester of pregnancy is not associated with increased adverse outcomes: results from the PIANO registry. Gastroenterology 2014;146:S-170.

13. Altaii H, Al-Kindi SG, Oliveira GH, et al. Aspirin use and risk of cholangiocarcinoma: External validation with big data. Hepatology 2017;65:1421-1422.

14. Alkhayyat M, Abou Saleh M, Aggrawal M, et al. Pancreatic manifestations in rheumatoid arthritis: a national populationbased study. Rheumatology (Oxford) 2020 Nov 27 [Online ahead of print]. doi: 10.1093/rheumatology/keaa616

15. Alkhayyat M, Abureesh M, Gill A, et al. Lower rates of colorectal cancer in patients with inflammatory bowel disease using AntiTNF therapy. Inflamm Bowel Dis 2020 Oct 14 [Online ahead of print]. doi: 10.1093/ibd/izaa252

16. Marmor S, Portschy PR, Tuttle TM, Virnig BA. The rise in appendiceal cancer incidence: 2000-2009. J Gastrointest Surg 2015; 19:743-750.

17. Hemminki K, Li X. Incidence trends and risk factors of carcinoid tumors: a nationwide epidemiologic study from Sweden. Cancer 2001;92:2204-2210.

18. Niederle MB, Hackl M, Kaserer K, Niederle B. Gastroenteropancreatic neuroendocrine tumours: the current incidence and staging based on the WHO and European Neuroendocrine Tumour Society classification: an analysis based on prospectively collected parameters. Endocr Relat Cancer 2010;17:909-918.

19. Taal BG, Visser O. Epidemiology of neuroendocrine tumours. Neuroendocrinology 2004;80 (Suppl 1): 1:3-7.

20. Ploeckinger U, Kloeppel G, Wiedenmann B, Lohmann R; representatives of 21 German NET Centers. The German NETregistry: an audit on the diagnosis and therapy of neuroendocrine tumors. Neuroendocrinology 2009;90:349-363.

21. Tsai HJ, Wu CC, Tsai CR, Lin SF, Chen LT, Chang JS. The epidemiology of neuroendocrine tumors in Taiwan: a nation-wide cancer registry-based study. PLoS One 2013;8:e62487.

22. O’Connor JM, Marmissolle F, Bestani C, et al. Observational study of patients with gastroenteropancreatic and bronchial neuroendocrine tumors in Argentina: Results from the large database of a multidisciplinary group clinical multicenter study. Mol Clin Oncol 2014;2:673-684.

23. Bayhan Z, Yildiz YA, Akdeniz Y, et al. Appendix neuroendocrine tumor: retrospective analysis of 4026 appendectomy patients in a single center. Emerg Med Int 2020;2020:4030527.

24. Mouhayar E, Salahudeen A. Hypertension in cancer patients. Tex Heart Inst J 2011;38:263-265.

25. Faggiano A, Ferolla P, Grimaldi F, et al. Natural history of gastroentero-pancreatic and thoracic neuroendocrine tumors. Data from a large prospective and retrospective Italian epidemiological study: the NET management study. J Endocrinol Invest 2012;35:817-823.

26. Kaerlev L, Teglbjaerg PS, Sabroe S, et al. The importance of smoking and medical history for development of small bowel carcinoid tumor: a European population-based case-control study. 
Cancer Causes Control 2002;13:27-34.

27. Rinzivillo M, Capurso G, Campana D, et al. Risk and protective factors for small intestine neuroendocrine tumors: a prospective case-control study. Neuroendocrinology 2016;103:531-537.

28. Hassan MM, Phan A, Li D, Dagohoy CG, Leary C, Yao JC. Risk factors associated with neuroendocrine tumors: A U.S.-based casecontrol study. Int J Cancer 2008;123:867-873.

29. Leoncini E, Carioli G, La Vecchia C, Boccia S, Rindi G. Risk factors for neuroendocrine neoplasms: a systematic review and metaanalysis. Ann Oncol 2016;27:68-81.

30. Hiripi E, Bermejo JL, Sundquist J, Hemminki K. Familial gastrointestinal carcinoid tumours and associated cancers. Ann Oncol 2009;20:950-954.

31. Basile U, Cavallaro G, Polistena A, et al. Gastrointestinal and retroperitoneal manifestations of type 1 neurofibromatosis. J Gastrointest Surg 2010;14:186-194.

32. Nadeem MS, Kumar V, Al-Abbasi FA, Kamal MA, Anwar F. Risk of colorectal cancer in inflammatory bowel diseases. Semin Cancer Biol 2020;64:51-60.

33. Grassia R, Bodini P, Dizioli P, et al. Neuroendocrine carcinomas arising in ulcerative colitis: coincidences or possible correlations?
World J Gastroenterol 2009;15:4193-4195.

34. West NE, Wise PE, Herline AJ, Muldoon RL, Chopp WV, Schwartz DA. Carcinoid tumors are 15 times more common in patients with Crohn's disease. Inflamm Bowel Dis 2007;13:1129-1134.

35. Freeman HJ. Appendiceal carcinoids in Crohn's disease. Can J Gastroenterol 2003;17:43-46.

36. Algaba A, Guerra I, Castaño A, et al. Risk of cancer, with special reference to extra-intestinal malignancies, in patients with inflammatory bowel disease. World J Gastroenterol 2013;19:93599365.

37. Khoudari G, Alkhayyat M, Abou Saleh M, et al. The epidemiology of pancreatic cancer and the association with acetylsalicylic acid in the United States: a population-based study. Pancreas 2020;49:1207-1212.

38. Khoudari G, Mansoor E, Click B, et al. Rates of intestinal resection and colectomy in inflammatory bowel disease patients after initiation of biologics: a cohort study. Clin Gastroenterol Hepatol 2020 Oct 14 [Online ahead of print]. doi: 10.1016/j.cgh.2020.10.008

39. Khan D, Abureesh M, Alkhayyat M, et al. Prevalence of myocardial infarction in patients with chronic pancreatitis. Pancreas 2021;50:99-103. 